\title{
Avaliação da neoformação óssea na sutura palatina mediana por meio de radiografia digitali- zada após a expansão assistida cirurgicamente*
}

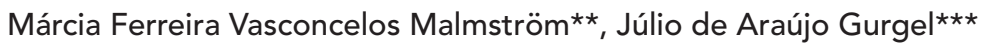

\begin{abstract}
Resumo
Objetivos: avaliar a neoformação óssea na região da sutura palatina mediana em pacientes adultos submetidos à expansão rápida da maxila assistida cirurgicamente. Metodologia: foram utilizadas radiografias oclusais convencionais periódicas obtidas da fase inicial e até 120 dias de contenção, digitalizadas em scanner com leitor de transparências, para a avaliação da densidade óptica. Resultados: a densidade óptica apresentou um aumento na fase final de contenção, porém, os valores de pixels obtidos em 120 dias foram significantemente inferiores aos valores da fase inicial. Conclusões: 1) os valores de pixels mostraram uma variabilidade individual no processo de remodelação da sutura; 2) não houve equiparação da densidade óptica no período final de contenção aos valores iniciais, demonstrando que o prazo de 120 dias não foi suficiente para a completa neoformação e remodelação óssea da sutura; 3 ) os resultados obtidos pelo método computadorizado corresponderam às informações obtidas nas radiografias oclusais convencionais, acrescentando a estas a análise quantitativa dos dados obtidos, podendo ser utilizado como método complementar de diagnóstico na prática clínica ortodôntica.
\end{abstract}

Palavras-chave: Expansão da maxila. Radiografia digital. Sutura palatina mediana.

\section{INTRODUÇÃO}

O período de contenção pós-expansão da sutura palatina mediana tem papel fundamental no tratamento ortodôntico, pois é neste período que ocorre a neoformação óssea e a remodelação da sutura após a disjunção, minimizando as possibilidades de recidiva $a^{7,16,18,19,26}$. O comportamento biológico da sutura palatina mediana, em pacientes adultos submetidos à expansão rápida da maxila assistida cirurgicamente (ERMAC), ainda não foi totalmente esclarecido, especialmente em relação ao período de contenção apropriado para que a área seja totalmente remodelada, visando maior estabilidade do tratamento.

A radiografia oclusal é o método de escolha para comprovar a abertura da sutura, para o controle do tratamento e o acompanhamento da neoformação óssea ${ }^{9,13}$. O processo de digitalização das radiografias possibilita a verificação da densidade radiográfica e a quantificação das alterações ocorridas, por meio das diferenças dos valores de pixels obtidos das imagens ${ }^{4,5}$.

** Mestre em Estomatologia e Especialista em Radiologia pela Faculdade de Odontologia de Bauru - USP. Professora de Radiologia e

Diagnóstico Bucal do curso de Odontologia da Universidade do Sagrado Coração - Bauru / SP.

*** Professor Titular de Ortodontia da Faculdade de Odontologia - UNICEUMA. Professor Assistentre Doutor da UNESP - Marília. 
Com o objetivo de avaliar a neoformação óssea na sutura palatina mediana em pacientes adultos submetidos à ERMAC, radiografias oclusais padronizadas foram realizadas periodicamente até 120 dias e digitalizadas por meio de um scanner com leitor de transparência. Os valores de pixels correspondentes à densidade óptica da área da sutura foram analisados utilizando-se um programa computadorizado de edição de imagens.

\section{REVISÃO DA LITERATURA}

O desenvolvimento de sistemas digitais exclusivos para uso em Odontologia e a difusão da Informática facilitaram o uso da radiografia digital, que vem sendo gradativamente adotada pelos profissionais da área da Radiologia, substituindo os filmes radiográficos convencionais, auxiliando no diagnóstico e possibilitando a realização de análises quantitativas ${ }^{15,30}$.

As vantagens da utilização da radiografia digital são a possibilidade de manipulação, melhoramento, armazenamento e intercâmbio das imagens para consultas e outros propósitos. Em adição a estes benefícios, existe a vantagem ambiental, diminuindo o uso de recursos naturais e reduzindo as doses de radiação $0^{30}$.

Com o crescente uso da imagem digital e das tecnologias disponíveis para a sua utilização nas áreas da saúde, o diagnóstico auxiliado por computador é uma ferramenta a mais para a obtenção de informações. Sendo o diagnóstico do radiologista baseado em uma avaliação subjetiva, e estando sujeito a variações intra e interpessoais, os registros armazenados no computador podem ser úteis na execução de análises quantitativas, eliminando o caráter de subjetividade, aumentando assim a precisão do diagnóstico ${ }^{5,30}$.

A expansão rápida da maxila tem sido utilizada eficazmente na correção das discrepâncias transversais maxilares em crianças e adolescentes até a fase puberal ${ }^{7,13,24}$. A separação dos ossos maxilares é obtida quando a força aplicada às estruturas dentoalveolares excede o limite necessário para a movimentação ortodôntica, antecipando a reação celular do ligamento periodontal e favorecendo a dissipação das forças para as suturas da maxila ${ }^{7,10}$. Em pacientes adultos, no entanto, há uma maior dificuldade na realização da expansão ortopédica da maxila, proporcionada pelo aumento da rigidez das estruturas craniofaciais, pela presença de sinostoses, as pontes ósseas na sutura palatina mediana, e pela maior resistência à distribuição das forças de expansão, causada especialmente pelos pilares nasomaxilar, zigomaticomaxilar e da junção pterigomaxilar ${ }^{6,9,17,23,24}$.

Embora este aumento da rigidez seja variável, oferece uma maior resistência às forças aplicadas à sutura durante a expansão maxilar, em pacientes que ultrapassaram o pico de crescimento puberal $1^{7,13,22}$. Para pacientes adultos e jovens, que já atingiram a maturidade esquelética, ou mesmo em pacientes que já tenham sido submetidos à terapia de expansão rápida maxilar sem sucesso, uma das terapias indicadas é a ERMAC 9,13,14,17,24,25.

Ten Cate, Freeman e Dickinson ${ }^{29}$, em 1977, avaliaram o desenvolvimento sutural em relação à estrutura e as respostas teciduais à expansão rápida da maxila, no intuito de identificar os elementos celulares, camadas constituintes da sutura e os eventos celulares seguintes à expansão rápida para a reconstituição da sutura. Os autores concluíram que as características da sutura, durante o desenvolvimento e quando submetida à expansão rápida, apresentam muitas similaridades. Além dos aspectos da inflamação inicial após a expansão rápida, a resposta da sutura é fibrogênese, osteogênese e remodelação, sendo estes últimos eventos observados de forma semelhante durante o período de crescimento.

Brin et al. ${ }^{11}$, em 1981, estudaram as respostas do osso na sutura palatina mediana após a aplicação de forças de tensão em animais jovens e idosos. As células examinadas neste estudo foram identificadas como osteoblastos e células osteoprogenitoras, classificadas com base em sua localização topográfica. Os autores concluíram que, 
em nível molecular, as células ósseas de animais idosos respondem à aplicação de forças mecânicas muito diferentemente das células de animais jovens. Esta diferença específica no conteúdo de nucleotídeos cíclicos das células formadoras de osso pode estar associada a uma inerente inabilidade dos osteoblastos de animais idosos em reconhecer estímulos externos ou agentes químicos gerados pelo estresse mecânico. Esses resultados sugerem, portanto, que diferenças entre indivíduos jovens e idosos em suas respostas à aplicação de forças aos dentes e maxilares podem estar intimamente associadas com eventos celulares específicos.

Em 1992, Souza ${ }^{28}$ avaliou o comportamento radiográfico, histológico e histométrico da sutura palatina mediana em macacos adultos submetidos à expansão maxilar. Após o período de 240 dias, os componentes da sutura apresentaram grande regeneração, definindo o novo processo ósseo. Os resultados mostraram que a regeneração da sutura ocorreu sem danos irreversíveis aos componentes e, radiograficamente, ao final do período de contenção estudado de oito meses, a sutura apresentava-se quase totalmente preenchida por formações radiopacas.

Em 1998, Kanekawa e Shimizu ${ }^{20}$ investigaram, em ratos, as alterações relacionadas à idade, durante a regeneração óssea na sutura palatina mediana, após a expansão maxilar. Os resultados mostraram que a dificuldade da expansão em ratos de 52 semanas e a diminuição da regeneração óssea após a expansão da sutura nos animais acima de 24 semanas podem ser relacionadas à idade, devido à maior rigidez esquelética, seguida pela diminuição da mineralização da matriz óssea. Segundo os autores, a sutura palatina mediana pode ser expandida nos casos que excedem o período puberal de crescimento, entretanto, deve-se ter maior atenção à neoformação óssea na sutura, pois - nestes casos - um tempo maior pode ser necessário para a suficiente mineralização da área.

Berger et al. ${ }^{8}$, em 1998, avaliaram comparativamente, em modelos de gesso e em radiografias póstero-anteriores, a estabilidade do tratamento utilizando expansão ortopédica e expansão rápida assistida cirurgicamente, em pacientes jovens e adultos. Os autores concluíram que ambas as técnicas mostraram resultados estáveis no período de contenção avaliado e que houve diferença estatisticamente significante na quantidade de expansão entre os grupos, sendo maior quando realizada a expansão assistida cirurgicamente. Destacaram a importância do período de contenção de um ano para o controle das forças peribucais na manutenção e estabilidade do tratamento.

Em 2003, Simões, Araújo e Bittencourt ${ }^{27}$ avaliaram a maturação óssea na região da sutura palatina mediana após expansão rápida da maxila em pacientes jovens. O objetivo deste estudo foi verificar a neoformação óssea durante os três meses de contenção do tratamento, avaliando o grau de densidade óptica na região da sutura por meio de imagem digitalizada, a partir de radiografias oclusais de pacientes submetidos à expansão maxilar. Em todas as áreas avaliadas houve uma neoformação óssea gradual, mas que ao final do período estudado ainda estava aquém dos valores iniciais.

Anttila et al. ${ }^{2}$, em 2004, avaliaram a viabilidade e estabilidade da expansão rápida da maxila assistida cirurgicamente com corticotomia lateral. O aparelho expansor foi mantido em posição, em média, por 6 meses (de 3 a 11 meses) para permitir a mineralização óssea. Os autores concluíram que a ERMAC pode ser realizada em pacientes acima de 30 anos com a técnica minimamente invasiva das paredes laterais maxilares, observaram também a grande variação individual em relação à presença de obliterações na sutura palatina mediana nos indivíduos adultos e que corticotomias adicionais poderiam ser necessárias em alguns casos, especialmente na região posterior.

\section{PROPOSIÇÃO}

Este estudo teve como objetivos:

1) avaliar o processo de neoformação óssea na região da sutura palatina mediana em pacientes 
adultos submetidos à ERMAC, por meio da densidade óptica das imagens radiográficas digitalizadas durante o período de contenção de 120 dias, comparando os valores de pixels obtidos das imagens nas diversas fases do estudo;

2) avaliar a aplicabilidade do método na prática clínica, como exame complementar para o acompanhamento da neoformação óssea da sutura palatina mediana submetida à expansão rápida.

\section{MATERIAL E MÉTODOS}

A amostra foi composta de 126 radiografias oclusais padronizadas, de 21 pacientes adultos submetidos à terapia de expansão rápida da maxila assistida cirurgicamente, realizadas nas fases inicial, pós-expansão e em 30, 60, 90 e 120 dias do período de contenção do tratamento.

Foram utilizados filmes radiográficos oclusais Insight da Kodak e um aparelho de raios X odontológico de $10 \mathrm{~mA}$ e $70 \mathrm{kV}$. As radiografias foram realizadas conforme a técnica oclusal total da maxila, com processamento manual dos filmes em câmara escura, pelo método temperatura/tempo, por um mesmo operador, seguindo as normas de biossegurança e radioproteção preconizadas.

Para a digitalização das radiografias utilizou-se um scanner de mesa ScanMaker 9800XL, fabricado nos Estados Unidos, pela Microtek International, 2003, com resolução óptica máxima de 1600 x 3200dpi, sensor CCD (Charge-coupled device), conversor A/D de 48 bits, equipado com um leitor de transparência (Transparent Media Adapter-TMA 1600) e seu respectivo programa para a captura da imagem, ScanWizard. Para a leitura da densidade óptica da imagem foi utilizado o programa editor de imagens Adobe Photoshop (Adobe, USA) e um microcomputador padrão IBM-PC, equipado com processador Pentium IV, 512MB de memória RAM, 3.00GHz, 80GB de espaço disponível no disco rígido, equipado com um monitor LG de 17", tela plana, modelo Flatron EZ T710SH (LG, USA), com resolução de tela de 1152 x 864 pixels e resolução de cores de 32 bits.
Após avaliação clínica e análise da documentação ortodôntica inicial, foram selecionados 21 pacientes adultos, sendo 14 femininos e 7 masculinos, brasileiros, com idades variando de 18 anos e 4 meses a 41 anos e 8 meses, sendo a média de 25 anos e 4 meses, com atresia maxilar e diferentes tipos de más oclusões, de acordo com os seguintes critérios:

1) deficiência transversal esquelética da maxila, cuja expansão maxilar justificasse a necessidade de efeito ortopédico, compatível com a indicação do procedimento cirúrgico; 2) presença de primeiros pré-molares superiores e primeiros ou segundos molares superiores, em ambos os hemiarcos, em condições estruturais e periodontais compatíveis com a cimentação e ativação do aparelho expansor; 3) condições fisiológicas do sistema cardiovascular favoráveis às intervenções cirúrgicas ambulatoriais.

Os pacientes foram devidamente informados, por escrito e verbalmente, sobre as condições da realização da pesquisa e um termo de consentimento livre e esclarecido foi assinado, concordando com a utilização das radiografias para esta pesquisa, a qual foi realizada segundo as normas estabelecidas pelo Comitê de Ética.

Foram utilizados aparelhos expansores dentosuportados do tipo Hyrax, com capacidade para 7 a $9 \mathrm{~mm}$ de expansão, confeccionados e instalados de acordo com as especificações contidas no protocolo estabelecido para a pesquisa, com bandas nos pré-molares e molares.

A fase cirúrgica foi realizada utilizando-se a técnica de expansão rápida da maxila assistida cirurgicamente, sob anestesia local. A ativação inicial de $2 \mathrm{~mm}$ do aparelho expansor foi realizada logo após a disjunção palatina. Após 48 horas da cirurgia prosseguiu-se com a ativação, sendo $2 / 4$ de volta de manhã e 2/4 à noite, até a obtenção da quantidade de expansão desejada, por um período de 7 dias. $\mathrm{O}$ aparelho disjuntor foi mantido em posição durante todo o período avaliado.

As radiografias oclusais convencionais foram 
examinadas por um radiologista, utilizando-se moldura em um negatoscópio, para melhor visualização dos detalhes da imagem. Foram avaliadas as características iniciais da sutura, a confirmação da disjunção, a presença de neoformação óssea e remodelação da área ao longo do período de estudo. As imagens foram observadas e comparadas quanto à morfologia e à radiopacidade nas diferentes fases do tratamento, especialmente as características da sutura palatina mediana da radiografia final em relação à radiografia inicial.

As radiografias foram digitalizadas em 600dpi, 8 bits, na escala de tons de cinza, e armazenadas no formato TIFF, sem compactação da imagem, na ordem de bits para PC-IBM. Para a captura das imagens utilizou-se uma moldura em cartolina preta, com a qual se padronizou a posição das radiografias sobre a mesa do scanner durante o procedimento de obtenção das imagens digitais.

Após a digitalização, realizou-se um recorte nas imagens, à direita e à esquerda, partindo do centro do parafuso expansor até suas margens laterais; superiormente, na margem da imagem radiográfica e, inferiormente, tangenciando as faces incisais dos incisivos superiores, gerando arquivos menores, com aproximadamente $820 \mathrm{~KB}$, onde a sutura palatina mediana aparece em toda sua extensão, centralizada na imagem, facilitando o arquivamento das imagens para leitura. Para a realização do recorte foi utilizada a ferramenta de Corte demarcado (Crop Tool).

Para a leitura da densidade óptica, representada pelos valores de pixels, nas áreas determinadas das radiografias digitalizadas, as imagens foram exibidas na tela do monitor em $100 \%$ do seu tamanho, facilitando a visualização, não sendo realizado qualquer tipo de ajuste na imagem, tais como bri1ho, contraste, etc, para a obtenção dos valores de pixels das áreas selecionadas.

Três áreas foram determinadas para as leituras, as quais foram demarcadas com a ferramenta Marca de seleção retangular (Rectangular markee too), com um tamanho fixo de 24 x 24 pixels, correspondente a uma superfície de $1 \mathrm{~mm}^{2}$, sobre a sutura palatina mediana. Determinou-se uma área localizada na região anterior e duas áreas na região posterior, sendo a primeira denominada $\mathrm{A}$, localizada na crista alveolar, entre os incisivos superiores; a segunda denominada $\mathrm{B}$, na região mais posterior da sutura, tangenciando a margem inferior do parafuso; e a terceira área denominda $\mathrm{C}$, lateralmente, à direita da segunda e à mesma altura. Nas radiografias pós-expansão, a área de leitura localizada na região anterior, área $\mathrm{A}$, foi determinada na metade da distância das cristas alveolares, as quais se encontravam separadas, bem como os incisivos superiores.

Após demarcada a área de leitura, o programa forneceu o histograma, demonstrando a média dos valores dos pixels contidos na área demarcada. Os valores apresentados são referentes à representação numérica dos pixels dentro da escala de cinza que varia de 0 (zero), representando a cor preta, a 255 , representando a cor branca. Os dados fornecidos no histograma foram então anotados em uma ficha elaborada para esta finalidade.

\section{ANÁLISE ESTATÍSTICA}

Os dados obtidos foram submetidos à análise estatística, por meio da análise de variância a um critério para medidas repetidas (ANOVA), teste de Tukey e análise de covariância. Para a verificação do erro intra-examinador foi utilizado teste $t$ pareado e a fórmula de Dahlberg.

\section{RESULTADOS E DISCUSSÃO}

O uso da imagem digital possibilita análises quantitativas das imagens radiográficas, pois transforma dados analógicos em dados numéricos, os quais podem ser analisados e comparados objetivamente. A sua utilização tem como finalidade aumentar a precisão na interpretação das imagens, uma vez que o diagnóstico radiográfico é subjetivo e dependente da experiência e conhecimento do profissional, da qualidade da imagem e das condições de visualização, fatores estes que podem 
comprometer a habilidade de visualização dos detalhes, a interpretação das imagens e a as decisões diagnósticas ${ }^{4,5,30}$.

Embora existam vários sistemas de radiografia digital direta disponíveis no $\operatorname{mercado}^{30}$, a radiografia digital indireta permanece como uma importante forma de se obter imagens digitais. Este método oferece a possibilidade de selecionar entre tamanhos de imagens, forma de arquivos, variação da escala de cinza e da resolução espacial. Possibilita também a digitalização de radiografias realizadas previamente, para que possam ser arquivadas digitalmente e utilizadas em estudos posteriores à sua realização $\mathrm{O}^{4,5,30}$.

A padronização é necessária, para que se minimize a ocorrência de variáveis que possam interferir no resultado da imagem radiográfica convencional final, tais como: variações de tempo e qualidade de exposição, devido às características próprias do aparelho radiográfico, como a miliamperagem e a kilovoltagem; erros de posicionamento, causando distorções e erros de processamento que poderiam promover alterações na densidade óptica da imagem, como super ou sub-revelação ${ }^{1}$.

As configurações do scanner para a digitalização das radiografias foram mantidas fixas para capturas em escala de cinza, com 8 bits, resultando em uma escala de 256 tons, variando de 0 a 255 , sendo os representativos numéricos da densidade óptica da radiografia. A vantagem da obtenção desta resolução de contraste é a possibilidade de identificação, pelo sistema computadorizado, de uma ampla gama de tonalidades de cinza, detectando sutis variações de densidade radiográfica, as quais dificilmente seriam diferenciadas pelo olho humano ${ }^{4,5,15,30}$.

A resolução espacial de captura das imagens de 600 dpi é adequada para que os detalhes da imagem sejam preservados, quando do processo de digitalização, possibilitando maior precisão diagnóstica. Esta resolução espacial é a mesma utilizada por alguns dos sistemas digitais diretos disponíveis atualmente no mercado ${ }^{4,29}$. Resolu- ções maiores podem não produzir imagens melhores, pois poderiam resultar na visualização dos cristais de prata contidos na emulsão do filme. Por outro lado, resoluções menores levariam à perda de informações da imagem original e limitariam a possibilidade de ampliação da imagem na tela do monitor, pois grandes ampliações tornam possíveis a visualização e diferenciação dos pixels que compõem a imagem digital, diminuindo a quantidade de detalhes e, conseqüentemente, a definição da imagem $^{15,30}$.

As características anatômicas da maxila e a resistência promovida pelas articulações laterais e superiores com os ossos craniofaciais determinam uma configuração piramidal da abertura entre os maxilares submetidos à expansão rápida. Esta abertura apresenta a base voltada para a região ântero-inferior e o fulcro localizado póstero-superiormente, em direção à cavidade nasal. A imagem observada na radiografia oclusal é a de uma área radiolúcida em forma de "V", onde a base está voltada para a região anterior, entre os incisivos centrais, geralmente separados por um diastema, evidenciando o maior espaço criado anteriormente, e o ápice voltado para a região posterior ${ }^{12,21,28}$. A seleção das três áreas de leitura foi feita baseada nas características da abertura e da remodelação da sutura palatina mediana.

Os resultados encontrados neste trabalho confirmaram os relatos da literatura, quanto à forma da abertura da sutura submetida às forças de expansão, que se deu em maior quantidade na região anterior em relação à região posterior. Foi visualizada, radiograficamente, uma ampla área radiolúcida entre as margens ósseas dos processos palatinos da maxila, em alguns casos de forma triangular bem definida e, em outros, de forma que as margens ósseas se encontravam mais paralelas, não sendo possível a visualização do vértice do triângulo na radiografia, denotando que a abertura se deu também em direção aos ossos palatinos.

A região anterior é relatada na literatura como a última região a completar a remodelação, sendo 
a área de abertura mais ampla do palato. A neoformação ocorre das margens ósseas em direção à linha média, tendo início na região posterior da

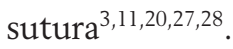

A área anterior A mostrou valores de pixels comparativamente menores que as outras duas áreas posteriores avaliadas, em todas as fases do tratamento, corroborando com as observações dos estudos prévios realizados com outras metodologias, pois estes valores indicaram a menor radiopacidade desta região. Estes dados são correspondentes aos dados obtidos pela análise radiográfica dos trabalhos de Melo $^{21}$; Simões, Araújo, Bittencourt ${ }^{27}$ e Souza ${ }^{28}$.

Analisando os resultados no gráfico 1, nota-se que a área $\mathrm{A}$, mesmo com valores de pixels menores em relação às outras duas áreas, apresentou um padrão de variação semelhante às áreas posteriores $\mathrm{B}$ e $\mathrm{C}$, quando as médias totais foram comparadas. Isto pode ser explicado pela localização da área $\mathrm{A}$ na margem do processo alveolar, onde observou-se o início do aumento da radiopacidade no sentido da extremidade inferior, restabelecendo primeiramente a área mais inferior do processo alveolar, em direção à região mais central da sutura.

Quando avaliadas as médias, pôde-se observar o padrão de variação da densidade óptica, como uma acentuada queda da densidade após a fase de ativação, correspondente à abertura da sutura palatina mediana, e um aumento gradual da radiopacidade no período de 30 dias. Houve um aumento

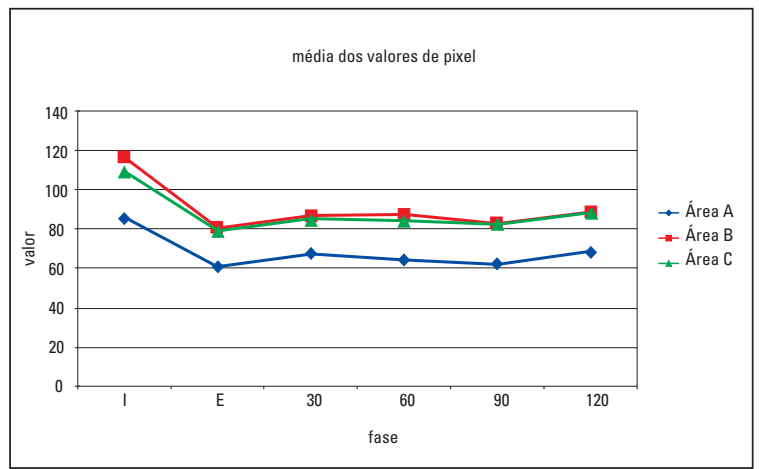

GRÁFICO 1 - Representação gráfica da distribuição das médias dos valores de pixels das áreas A, B e C, nas fases inicial (I), pós-expansão (E), contenção de 30, 60, 90 e 120 dias. dos valores a partir de 90 dias até 120 dias, entretanto, as densidades dos pixels obtidas no final do período de estudo não alcançaram os valores do início do tratamento. Os valores de pixels finais foram 20,1\% menores que os iniciais para a área A, 23,7\% e 19,34\% menores para as áreas B e C, respectivamente.

Variações individuais foram observadas durante a análise visual das imagens radiográficas, onde foram detectadas diferenças na quantidade e na forma da abertura da sutura palatina mediana, assimetrias e presença de fragmentos e espículas ósseas na região e, ainda, momentos diferenciados de neoformação óssea durante a remodelação da área. Estas características diferenciadas da abertura da sutura palatina mediana também foram observadas em estudos prévios ${ }^{7,28}$.

Mesmo diante de observações radiográficas das diferentes respostas entre os indivíduos da amostra, os resultados estatísticos não foram significantes, quando analisadas as relações entre a idade e o gênero dos pacientes pela análise de covariância. Isto talvez possa ser explicado pela faixa etária selecionada, sendo a amostra avaliada neste estudo composta somente por pacientes adultos, não tendo sido comparada com outra amostra de outra faixa etária, e pelo número de pacientes dos grupos feminino $(n=14)$ e masculino $(n=7)$. Estes resultados são semelhantes aos resultados encontrados por $\mathrm{Melo}^{21}$, os quais não demonstraram diferenças estatisticamente

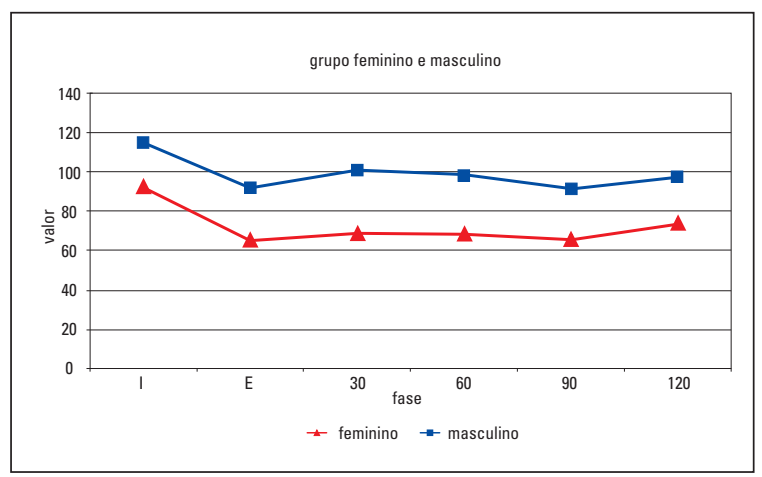

GRÁFICO 2 - Representação gráfica das médias dos valores de pixe/s das áreas A, B e C nas fases inicial (I), pós-expansão (E) e contenção de 30, 60, 90 e 120 dias, dos grupos feminino e masculino. 
significantes em relação à idade e ao gênero, quando avaliada uma amostra composta apenas por pacientes jovens.

Pela análise das imagens digitalizadas, pôde-se observar e quantificar a diferença entre as médias totais dos valores de pixels dos grupos feminino e masculino, representativos da maior densidade óssea nos pacientes do gênero masculino (Gráf. 2), característica dificilmente detectada pela análise visual e subjetiva realizada pelo profissional.

Quanto aos aspectos de mineralização da sutura, os achados radiográficos se assemelham aos resultados encontrados por Arat et al. ${ }^{3}$; Belli7; Ekström, Henrikson, Jensen ${ }^{16}$; Ten Cate, Freeman e Dickinson ${ }^{29}$ pelo padrão de neoformação óssea em sentido perpendicular a partir das margens ósseas, em direção ao centro e início na região posterior em direção à porção média da sutura, e por último a mineralização ocorre na região anterior e média. $\mathrm{O}$ aumento gradual da radiopacidade na região da sutura expandida ao longo do período de contenção, também concorda com os resultados obtidos por estes autores.

As características da remodelação observadas durante a análise visual das radiografias foram descritas e podem ser visualizadas nas figuras 1 a 6 .

\section{RESULTADOS DA ANÁLISE VISUAL DAS RA- DIOGRAFIAS OCLUSAIS CONVENCIONAIS}

Da análise visual das imagens radiográficas, um padrão geral pôde ser observado nas diferentes fases de tratamento avaliadas.

\section{Inicial (Fig. 1)}

Características das suturas: variações existentes, quando observado o espaço radiolúcido entre as margens ósseas, entre suturas retilíneas, sinuosas, interdigitadas e pouco visíveis.

\section{Pós-expansão (Fig. 2)}

Visualização da abertura: o padrão observado foi o da abertura como uma área radiolúcida de forma triangular, com a base voltada para a região entre os incisivos centrais e vértice voltado para posterior, algumas suturas mostraram padrão de abertura mais paralelo.

Margens ósseas: algumas bem definidas e outras apresentaram fragmentos radiopacos ligados ou próximos às margens ósseas, ou dentro da área expandida.

\section{0 dias (Fig. 3)}

Característica da abertura: aumento da área radiolúcida em relação à radiografia anterior, tanto em largura quanto em comprimento. Algumas suturas apresentaram aberturas mais paralelas. Ligeiro aumento da radiopacidade de forma difusa em toda a extensão da sutura.

Margens ósseas: menor definição das margens e dos fragmentos radiopacos, quando presentes.

\section{0 dias (Fig. 4)}

Margens ósseas: tornam-se menos definidas. Apenas em um paciente as corticais antigas foram mantidas e radiograficamente são visualizadas como linhas de maior radiopacidade ao longo da área da abertura, bilateralmente.

Neoformação óssea: em algumas radiografias já é possível a visualização de áreas com aumento da radiopacidade, especialmente na área posterior e próximas às margens ósseas. Radiopacidade difusa semelhante à fase anterior ao longo da área expandida.

\section{0 dias (Fig. 5)}

Neoformação óssea: a mineralização nas margens ósseas já se torna mais evidente, com aspectos de "raios" no sentido transversal, perpendicular às antigas margens ósseas da sutura. Maior aumento de radiopacidade na porção posterior e nas margens laterais. Início do aumento da radiopacidade na crista alveolar entre os incisivos superiores.

\section{0 dias (Fig. 6)}

Neoformação óssea: progressão da mineralização, aumento gradual da radiopacidade da região 


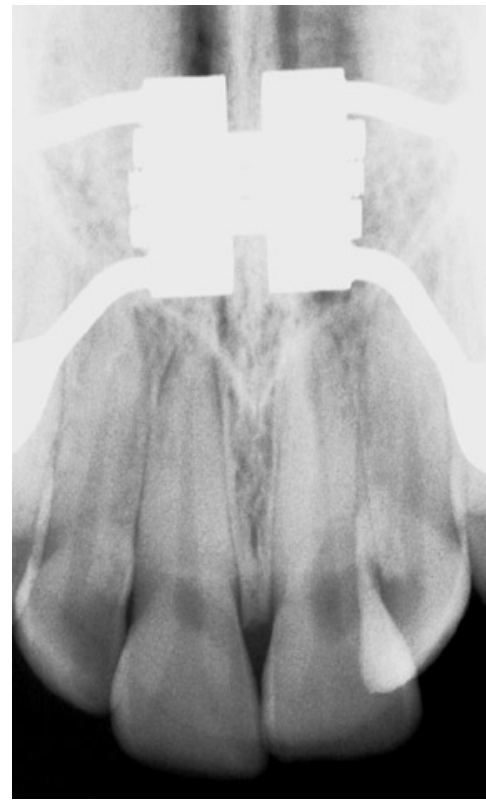

FIGURA 1 - Inicial.

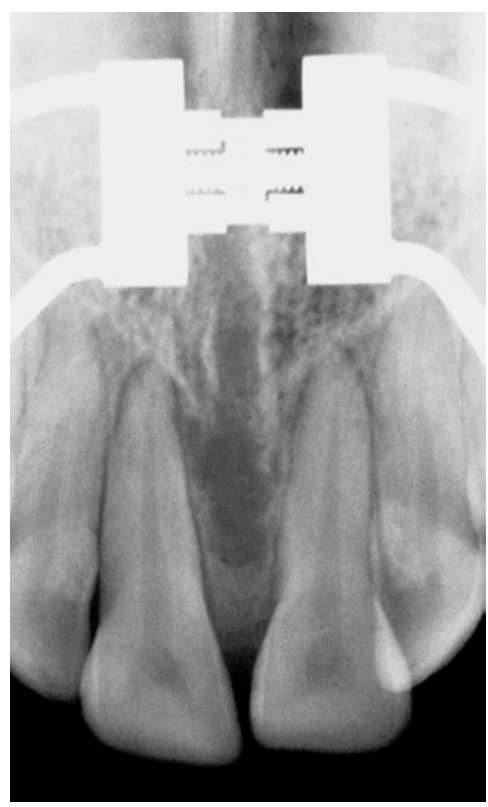

FIGURA 4 - 60 dias.

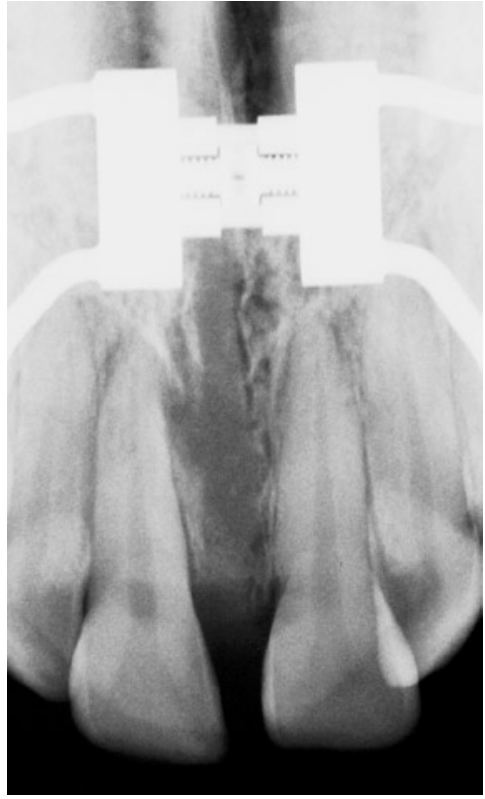

FIGURA 2 - Pós-expansão.

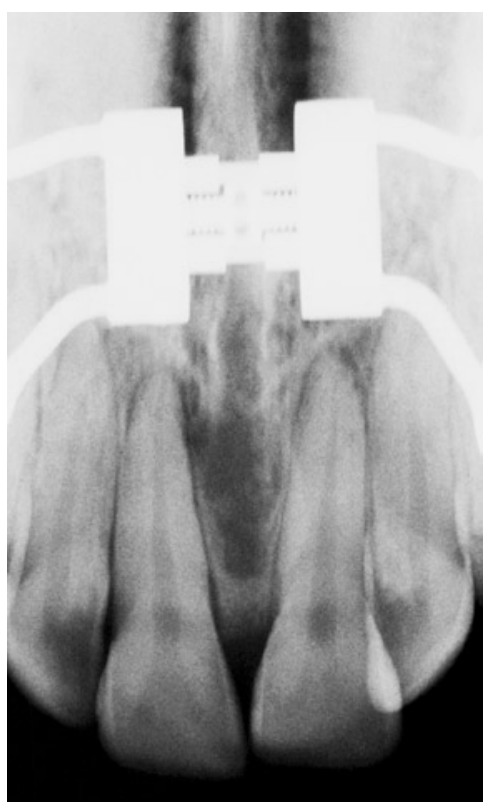

FIGURA 5 - 90 dias

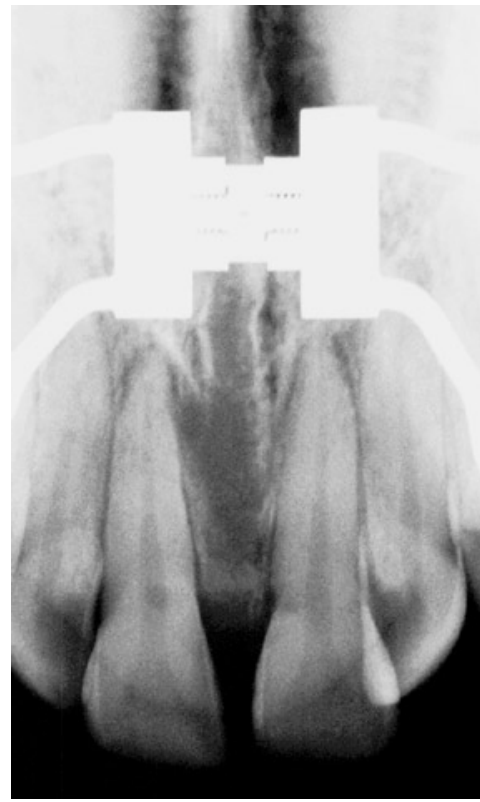

FIGURA 3 - 30 dias.

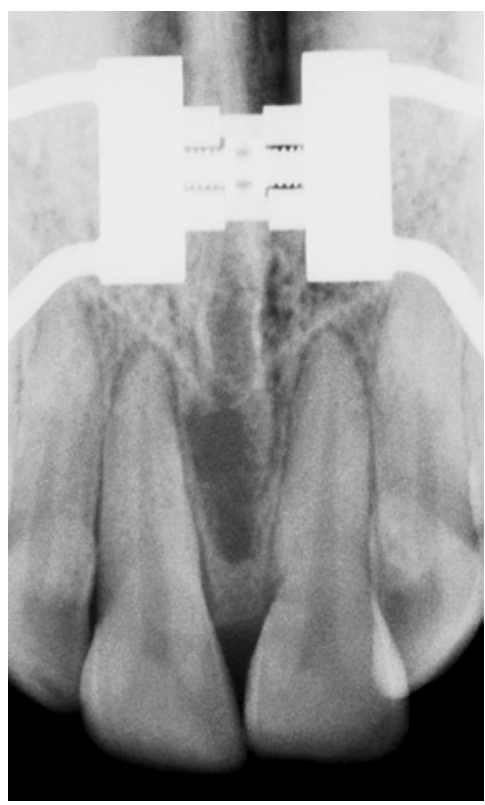

FIGURA 6 - 120 dias. posterior em direção à região média da sutura, das margens ósseas em direção ao centro e início da remodelação da crista alveolar, próximo às raízes dos incisivos superiores. A área central da sutura apresenta pouca radiopacidade, comparada à radiografia inicial.
Margens ósseas: ainda apresentam aspecto difuso, sem definição das margens. Nota-se a redução do diastema.

Da comparação dos valores de pixels das áreas avaliadas nas diversas fases do tratamento, foi constatado que em nenhum dos pacientes ocorreu 
a equiparação aos valores da imagem inicial durante o período de acompanhamento de 120 dias. Esta diferença dos valores da densidade óptica foi significativa, com $\mathrm{p}<0,05$ para todas as outras áreas, como demonstrado estatisticamente (Tab. 1).

Estes dados se diferenciam de outros estudos realizados em pacientes ou modelos experimentais jovens, onde se constatou que em três meses de contenção houve completa remineralização e remodelação da área da sutura ${ }^{16,21,28}$, possivelmente pela diferenciação das idades dos indivíduos das amostras estudadas, influenciando o processo de remodelação da sutura. Este, em indivíduos adultos e idosos, é retardado quando comparado com indivíduos jovens e crianças ${ }^{3,11,20}$.

De modo geral, é recomendado um período de contenção fixa, mantendo o próprio expansor em posição, por três meses e mais seis meses de contenção com a placa removível ou barra transpalatina ${ }^{7,9,10,14}$. Este protocolo pode ser o suficien- te para pacientes jovens e crianças, porém os resultados encontrados mostraram que, em até 120 dias de contenção, não houve mineralização óssea suficiente para o restabelecimento da densidade óptica e da arquitetura inicial da sutura palatina mediana nos pacientes adultos avaliados.

Os resultados encontrados neste estudo, talvez, possam auxiliar na compreensão das recidivas clínicas ocorridas em pacientes adultos, quando submetidos a períodos de contenção de até três meses, e atribuídas à parte ortodôntica, ao passo que este período mostrou não ser o suficiente para a manutenção do ganho ortopédico obtido durante a expansão maxilar e ajustes fisiológicos de adaptação à nova posição das estruturas envolvidas.

Estes dados nos levam a sugerir que, para os pacientes adultos tratados com expansão rápida maxilar assistida cirurgicamente, o período de contenção seja monitorado e estendido, respeitando-se as variações individuais, no que se refere à

Tabela 1 - Análise de variância (ANOVA), teste de Tukey, valores mínimos e máximos das áreas A, B e C nas fases inicial (I), pós-expansão (E), contenção de 30, 60, 90 e 120 dias e os valores das médias e desvios-padrão.

\begin{tabular}{|c|c|c|c|c|c|c|}
\hline área & ANOVA & Tukey & mínimo & máximo & $\overline{\mathbf{x}}$ & d.p. \\
\hline Al & \multirow{6}{*}{$\begin{array}{c}F=9,8 \\
p<0,05^{*}\end{array}$} & Al & 45 & 112 & 85,6 & 16,9 \\
\hline $\mathrm{AE}$ & & 0,00012 * & 26 & 87 & 60,9 & 17,1 \\
\hline A30 & & 0,00045 * & 34 & 102 & 67,6 & 18,5 \\
\hline A60 & & $0,00013 *$ & 22 & 96 & 64,2 & 17,8 \\
\hline A90 & & 0,00012 * & 34 & 101 & 62,2 & 15,9 \\
\hline $\mathrm{A} 120$ & & $0,00089 *$ & 34 & 104 & 68,4 & 20,3 \\
\hline $\mathrm{BI}$ & \multirow{6}{*}{$\begin{array}{l}F=17,1 \\
p<0,05 *\end{array}$} & $\mathrm{Bl}$ & 81 & 142 & 116,0 & 20,4 \\
\hline $\mathrm{BE}$ & & $0,00012 *$ & 35 & 125 & 80,7 & 24,4 \\
\hline B30 & & 0,00012 * & 35 & 140 & 86,5 & 24,7 \\
\hline $\mathrm{B} 60$ & & 0,00012 * & 55 & 148 & 87,0 & 25,2 \\
\hline B90 & & 0,00012 * & 45 & 141 & 82,7 & 23,7 \\
\hline B120 & & 0,00012 * & 44 & 131 & 88,5 & 22,2 \\
\hline $\mathrm{Cl}$ & \multirow{6}{*}{$\begin{array}{l}F=11,4 \\
p<0,05^{*}\end{array}$} & $\mathrm{Cl}$ & 65 & 142 & 109,1 & 21,8 \\
\hline CE & & 0,00012 * & 35 & 115 & 79,0 & 21,2 \\
\hline C30 & & 0,00012 * & 36 & 126 & 84,9 & 22,3 \\
\hline $\mathrm{C} 60$ & & 0,00012 * & 40 & 151 & 84,1 & 25,7 \\
\hline C90 & & 0,00012 * & 35 & 141 & 82,5 & 24,1 \\
\hline C120 & & 0,00024 * & 35 & 124 & 88,0 & 21,9 \\
\hline
\end{tabular}

* estatisticamente significante. 
neoformação e maturação ósseas e remodelação da área expandida ortopedicamente, maximizando assim os resultados e a estabilidade do tratamento, até que a área da sutura expandida esteja totalmente mineralizada.

Diante dos resultados obtidos e suas implicações na prática ortodôntica, observa-se a necessidade de um estudo em longo prazo, com o objetivo de avaliar qual o período ideal de contenção pós-expansão rápida da maxila em pacientes adultos, para que a remodelação da sutura se complete, possibilitando a reorganização dos tecidos e das estruturas envolvidas no tratamento, minimizando as indesejadas recidivas clínicas.

O segredo do sucesso da terapia ortodôntica em adultos reside nas considerações das características próprias da idade, dos princípios histológicos e fisiológicos, os quais auxiliam na determinação do plano de tratamento, especialmente nos períodos de contenção $\mathrm{o}^{2,3,7,9,11,19,20,25}$.

\section{CONCLUSÕES}

1) Os valores de pixels nas radiografias digitalizadas apresentaram variações nas fases pósexpansão imediata e de contenção do tratamento, representando a variabilidade individual no processo de remodelação da sutura palatina mediana entre os pacientes.
2) A densidade óptica, representada pelos valores de pixels, apresentou um aumento ao final do período de contenção de 120 dias, porém, não houve equiparação destes valores aos valores da fase inicial, demonstrando que este prazo não foi o suficiente para o restabelecimento da densidade óptica e completa neoformação óssea na sutura nos pacientes adultos avaliados.

3) Os resultados obtidos pelo método digitalizado de imagens foram correspondentes aos resultados da análise visual das radiografias convencionais, possibilitando a análise quantitativa dos dados obtidos, podendo assim ser utilizado como método complementar na prática clínica.

\section{AGRADECIMENTOS}

Os autores agradecem aos cirurgiões-dentistas Luiz Fernando de Mello Santana e Fernando Paganeli Machado Giglio, alunos do curso de Doutorado do Programa de Pós-graduação em Estomatologia da Faculdade de Odontologia de Bauru da Universidade de São Paulo, pela realização da fase cirúrgica deste trabalho.

\title{
Evaluation of new bone formation at the midpalatal suture by digitized radiography after surgically assisted maxillary expansion
}

\begin{abstract}
Objectives: To evaluate the new bone formation at the midpalatal suture in adult patients submitted to surgically assisted rapid maxillary expansion. Methods: Regular conventional occlusal radiographs were obtained from the initial stage up to 120 days and digitized with a scanner with transparency adapter for evaluation of optical density. Results: The optical density presented an increase at the final stage of retention, yet the pixe/values achieved at 120 days were significantly lower than the values of the initial stage. Conclusions: 1) the pixe/values exhibited an individual variability in the process of suture remodeling; 2 ) there was no similarity of optical density between the final period of retention and the initial values, demonstrating that the 120-day period was not enough for complete new bone formation and bone remodeling at the suture; 3 ) the results achieved by the computerized method corresponded to the information achieved on the conventional occlusal radiographs, adding the quantitative analysis of the obtained data, and may be used as a complementary diagnostic method in the orthodontic clinic.
\end{abstract}

Key words: Rapid maxillary expansion. Digital radiography. Midpalatal suture. 


\section{REFERÊNCIAS}

1. ALVARES, L. C.; TAVANO, O. Curso de radiologia em Odontologia. 4. ed. São Paulo: Ed. Santos, 2002.

2. ANTTILA, A. et al. Feasibility and long-term stability of surgical assisted rapid maxillary expansion with lateral osteotomy. Eur. J. Orthod., Oxford, v. 26, no. 4, p. 391-395, Aug. 2004.

3. ARAT, Z. M. et al. $99 \mathrm{mTechnetium-labeled} \mathrm{methylene} \mathrm{diphos-}$ phonate uptake in maxillary bone during and after rapid maxillary expansion. Angle Orthod., Appleton, v. 73, no. 5, p. 545-549, Oct. 2003.

4. ATTAELMANAN, A.; BORG, E.; GRÖNDHAL, H. G. Digitisation and display of intra-oral films. Dentomaxillofac. Radiol., Houndsmills, v. 29, no. 2, p. 97-102, Mar. 2000.

5. AZEVEDO-MARQUES, P. M. Diagnóstico auxiliado por computador na Radiologia. Radiol. Bras., v. 34, no. 5, p. 285-293, 2001.

6. BACCETTI, T. et al. Treatment timing for rapid maxillary expansion. Angle Orthod., Appleton, v. 71, no. 5, p. 343-350, Oct. 2001.

7. BELL, R. A. A review of maxillary expansion in relation to rate of expansion and patient's age. Am. J. Orthod., St. Louis, v. 81, no. 1, p. 32-37, Jan. 1982

8. BERGER, J. L. et al. Stability of orthopedic and surgically assisted rapid palatal expansion over time. Am. J. Orthod. Dentofacial Orthop., St. Louis, v. 114, no. 6, p. 638-645, Dec. 1998.

9. BETTS, N. J. et al. Diagnosis and treatment of transverse maxillary deficiency. Int. J. Adult Orthodon. Orthognath Surg., Chicago, v. 10, no. 2, p. 75-96, 1995.

10. BISHARA, S. E.; STALEY, R. N. Maxillary expansion: clinical implications. Am. J. Orthod. Dentofacial Orthop., St. Louis, v. 91, no. 1, p. 3-14, Jan. 1987.

11. BRIN, I. et al. Rapid palatal expansion in cats: effect of age on sutural cyclic nucleotides. Am. J. Orthod., St. Louis, v. 79, no. 2, p. 162-175, Feb. 1981.

12. BROSH, T. et al. Rapid palatal expansion. Part 3: strains developed during active and retention phases. Am. J. Orthod. Dentofacial Orthop., St. Louis, v. 114, no. 2, p. 123-133, Aug. 1998.

13. CAPELOZZA FILHO, L.; SILVA FILHO, O. G. Expansão rápida da maxila: considerações gerais e aplicação clínica. Parte I. Rev. Dental Press Ortodon. Ortop. Facial, Maringá, v. 2, n. 3, p. 88-104, maio/jun. 1997.

14. CAPELOZZA FILHO, L.; SILVA FILHO, O. G. Expansão rápida da maxila: considerações gerais e aplicação clínica. Parte II. Rev. Dental Press Ortodon. Ortop. Facial, Maringá, v. 2, n. 4, p. 86-108, jul./ago. 1997

15. CHEN, S. K.; HOLLENDER, L. Digitizing of radiographs with a flatbed scanner. J. Dent., Bristol, v. 23, no. 4, p. 205-208, 1995.

16. EKSTRÖM, C.; HENRIKSON, C. O.; JENSEN, R. Mineralization in the midpalatal suture after orthodontic expansion. Am. J. Orthod., St. Louis, v. 71, no. 4, p. 449-455, Apr. 1977.

17. GLASSMAN, A. S. et al. Conservative surgical orthodontic adult rapid palatal expansion: sixteen cases. Am. J. Orthod., St. Louis, v. 86, no. 3, p. 207-213, Sept. 1984.

18. HAAS, A. J. Long-term post treatment evaluation of rapid palatal expansion. Angle Orthod., Appleton, v. 50, no. 3, p. 189-217, July 1980 .

19. KAHL-NIEKE, B. Retention and stability considerations for adult patients. Dent. Clin. North Am., Philadelphia, v. 40, no. 4, p. 961-992, Oct. 1996.

20. KANEKAWA, M.; SHIMIZU, N. Age-related changes on bone regeneration in midpalatal suture during maxillary expansion in the rat. Am. J. Orthod. Dentofacial Orthop., St. Louis, v. 114, no. 6, p. 646-653, Dec. 1998.

21. MELO, M. F. B. Avaliação da densidade ótica da sutura palatina mediana por meio do sistema de radiografia digital por placa fotoativada em pacientes submetidos à disjunção palatina. 2003. 88 f. Tese (Doutorado)-Faculdade de Odontologia, Universidade de São Paulo, São Paulo, 2003.
22. MELSEN, B. Palatal growth studied on human autopsy material: a histologic microradiographic study. Am. J. Orthod., St. Louis, v. 68, no. 1, p. 42-54, July 1975.

23. NORTHWAY, W. M.; MEADE JR., J. B. Surgically assisted rapid maxillary expansion: a comparison of technique, response, and stability. Angle Orthod., Appleton, v. 67, no. 4, p. 309-320, 1997.

24. POGREL, M. A. et al. Surgically assisted rapid maxillary expansion in adults. Int. J. Adult Orthodon. Orthognath Surg., Chicago, v. 7, no. 1, p. 37-41, 1992.

25. PROFFIT, W. R.; TURVEY, T. A.; PHILLIPS, C. Orthognathic surgery: a hierarchy of stability. Int. J. Adult Orthodon. Orthognath Surg., Chicago, v. 11, no. 3, p. 191-204, 1996.

26. SILVA FILHO, O. G. et al. Expansão rápida da maxila: um ensaio sobre a sua instabilidade. Rev. Dental Press Ortodon. Ortop. Facial, Maringá, v. 8, n. 1, p. 17-36, jan./fev. 2003.

27. SIMÕES, F. X. P. C.; ARAÚJO, T. M. A.; BITTENCOURT, M. A. V. Avaliação da maturação óssea na região da sutura palatina mediana, após expansão rápida da maxila, por meio de imagem digitalizada. Rev. Dental Press Ortodon. Ortop. Facial, Maringá, v. 8, n. 1, p. 59-67, jan./fev. 2003.

28. SOUZA, M. M. G. Comportamento radiográfico, histológico e histométrico da sutura palatina mediana de primatas adultos (Cebus apella) submetidos à expansão maxilar 1992. 143 f. Dissertação (Mestrado)-Faculdade de Odontologia, Universidade Federal do Rio de Janeiro, Rio de Janeiro, 1992.

29. TEN CATE, A. R.; FREEMAN, E.; DICKINSON, J. B. Sutural development: structure and its response to rapid expansion. Am. J. Orthod., St. Louis, v. 71, no. 6, p. 622-636, June 1977.

30. VAN DER STELT, P. F. Principles of digital imaging. Dent. Clin. North Am., Philadelphia, v. 44, no. 2, p. 237-248, Apr. 2000.

\section{Endereço para correspondência}

Márcia Ferreira Vasconcelos Malmström

Rua Dr. Sérvio T. Carrijo Coube, 3-33, Ap. 173B

Jd. Infante D. Henrique

CEP: 17.012-632 - Bauru/SP

E-mail: marciamalmstrom@gmail.com 